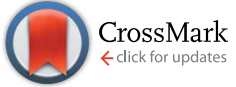

Cite this: RSC Adv., 2015, 5, 29135

Received 16th January 2015 Accepted 18th March 2015

DOI: $10.1039 / c 5 r a 00964 b$

www.rsc.org/advances

\section{Cell uptake mechanisms of glycosylated cationic pDNA-cyclodextrin nanoparticles}

\author{
Nicolas Guilloteau, ${ }^{a}$ Céline Bienvenu, ${ }^{a}$ Coralie Charrat, ${ }^{a}$ José L. Jiménez Blanco, ${ }^{b}$ \\ Alejandro Díaz-Moscoso, ${ }^{c}$ Carmen Ortiz Mellet, ${ }^{b}$ José M. García Fernández, ${ }^{* c}$ \\ Pierre Vierling ${ }^{a}$ and Christophe Di Giorgio*a
}

\begin{abstract}
The incorporation of carbohydrate functional elements in the architecture of polycationic amphiphilic cyclodextrins (paCDs) provides glycosylated paCDs ( $p G a C D s$ ) that form transfectious nanocomplexes (glycoCDplexes) with pDNA. In this study, we aimed at elucidating the internalization mechanisms at play and their incidence in transfection efficiency for glycoCDplexes formulated with 6-amino-6-deoxy- $\beta$-Dglucopyranosyl-appended pGaCDs in comparison with mannosylated and non-glycosylated congeners. Preliminary data showed a relatively high uptake of the 6 -aminoglucosylated nanocomplexes by BNLCL2 hepatocytes that correlated with a strong affinity towards the galactose-specific peanut agglutinin (PNA) lectin, suggesting that the galactose-binding asialoglycoprotein receptor at the surface of hepatocytes might be involved in glycoCDplex internalization. Transfection kinetics, internalization rates and protein expression data in BNL-CL2 ASGPR-expressing cells and COS-7 ASGPR-devoid epithelial cells in the absence and presence of different inhibitors of clathrin-dependent (chlorpromazine), caveolae-dependent (genistein) and macropinocytosis (amiloride) endocytic routes evidenced significant differences in cell uptake pathways and fate of glycoCDplexes as compared with CDplexes. Most importantly, such differences were dependent on the cell type and on the carbohydrate coating moiety. Clathrin-mediated uptake in BNLCL-2 cells is particularly favored for the 6-amino-6-deoxyglucose CDplexes, supporting the interplay of specific recognition phenomena. Competitive uptake and transfection experiments conducted in the presence of asialofetuin or of a polyclonal ASGPR-antibody, as well as siRNA-mediated ASGPR-specific gene knockdown, supported the involvement of ASGPR, firmly demonstrating the dual role of the 6-amino-6-deoxyglucose motif as DNA and lectin receptor ligand. The results reinforce the use of carbohydrates in glycoCDplexes to modulate cellular uptake and transfection capabilities in a cell-dependent manner.
\end{abstract}

\section{Introduction}

Clinical translation of gene delivery into a form of molecular therapy that addresses a medical problem at its source progresses slowly largely due to the absence of efficient and safe carrier systems (vectors) enabling the nucleic acid cargo (DNA, siRNA, miRNA, mRNA, oligonucleotides, aptamers, DNAzymes) to reach its target. ${ }^{1,2}$ The use of disarmed recombinant viral vectors remains as the most efficient method of promoting genetic material uptake and expression at present. ${ }^{3}$ However, the risk of immunogenicity, residual infectivity and insertional

${ }^{a}$ Institut de Chimie de Nice, UMR 7272, Université de Nice Sophia Antipolis, CNRS, 28, Avenue de Valrose, F-06108 Nice, France. E-mail: christophe.di-giorgio@unice.fr; Fax: +33 492076151; Tel: +33492076158

${ }^{b}$ Departamento de Química Orgánica, Facultad de Química, Universidad de Sevilla, c/ Profesor García González 1, E-41012 Sevilla, Spain

'Instituto de Investigaciones Quimicas (IIQ), CSIC - Universidad de Sevilla, Américo Vespucio 49, Isla de la Cartuja, E-41092 Sevilla, Spain.E-mail: jogarcia@iiq.csic.es; Fax: +34 954460165; Tel: +34954489553 mutagenesis has tempered the initial prospects and currently precludes their widespread application. Alternatively, non-viralbased vectors have gathered momentum. ${ }^{4,5}$ A large diversity of biocompatible materials has been produced during the last three decades, most of which fall within the category of cationic lipids $s^{6,7}$ or polymers, ${ }^{\mathbf{8} 9}$ with the capability to compact DNA into transfectious nanoparticles (lipoplexes and polyplexes, respectively). Molecular facial amphiphiles, featuring segregated multihead (polycationic) and multitail (hydrophobic) domains separated by a nanometric platform have emerged as a new generation of non-viral vectors. ${ }^{\mathbf{1 0 - 1 3}}$ Among them, polycationic amphiphilic cyclodextrins (paCDs) have proven particularly promising both in vitro ${ }^{\mathbf{1 4 - 1 9}}$ and in vivo. ${ }^{\mathbf{2 0}}$ Their monodisperse character, truncated cone-shaped topology and availability in three different sizes, namely the hexa- $(\alpha C D)$, hepta- $(\beta C D)$ or octameric- $(\gamma \mathrm{CD})$ cyclomaltooligosaccharide platform, allows a precise structural and conformational control and is compatible with the incorporation of functional elements, e.g. for targeting, ${ }^{21-25}$ with a relatively low synthetic cost. However, 
although transfection efficiencies that rival those of the most popular commercial non-viral gene vectors, such as JetPEI ${ }^{\circledR}$ or LipofectAmine $2000 \AA$, have been achieved, their performance is still far from that of the viral counterparts, which limits their application range.

Progress in the field of gene therapy requires a better understanding of the mechanisms whereby vector:pDNA complexes overcome transfection barriers to then induce transgene expression, followed by implementation of that knowledge in vector design. It has become increasingly evident that the way the DNA nanoparticles are taken up by the cells has a direct consequence in intracellular trafficking and ultimately in gene expression, some pathways being more productive than others in this respect. ${ }^{26-29}$ All these investigations have further shown that the uptake mechanism of DNA nanoparticles is dependent upon the cell type, the nanoparticle size, and/or the chemical nature of the DNA condensing agent. In the case of paCD:DNA nanocomplexes (CDplexes), a preliminary study carried out with formulations containing the dendritic $\beta C D$ derivative 1 (Fig. 1) in kidney epithelial Vero cells indicated that they were mainly internalized by clathrin-dependent endocytosis and, to a lesser extent, by caveolin-mediated endocytosis, ${ }^{30}$ the latter pathway being predominantly responsible for successful transfection. In any case, no cell-specific internalization mechanisms were foreseen.

A further comparative study using CDplexes formulated with the tetradecacationic amphiphilic $\beta$-cyclodextrin derivative 2, one of the better-performing paCD representatives reported up to date, and the two glycosylated polycationic amphiphilic CDs (gpaCDs) 3 and 4 (Fig. 1) demonstrated the potential of glycotargeting for site-specific gene delivery. ${ }^{22,31,32}$ Thus, CDplexes obtained from gpaCD prototype 4 , bearing seven primary amino groups and seven $\alpha$-D-mannopyranosyl ( $\alpha$-Man) glycotopes in alternate branches (ManCDplexes), were shown to be preferentially uptaken in macrophages through the macrophage mannose receptor (MMR), in agreement with the sugar specificity of this lectin. Strikingly, control experiments using other cell lines and lectin receptors evidenced a comparatively high uptake of CDplexes formulated with gpaCD 3, incorporating 6-amino-6-deoxy- $\beta$-D-glucopyranose substituents (GluCDplexes), in BNL-CL2 (mouse normal embryonic hepatocytes) cells expressing the asialoglycoprotein receptor (ASGPr), a C-type lectin that plays a central role in the clearance of circulating glycoproteins exposing galactopyranosyl residues. $^{33}$ These data raised the question whether the 6-amino-6-deoxy- $\beta$ D-glucopyranosyl substituent (a motif present in aminoglycoside antibiotics known to interact with nucleic acids ${ }^{34}$ ) behaves as a galactose surrogate with multimodal binding capabilities towards membrane receptors. Motivated by the promise of such a functional element in gene vector design strategies, we have investigated the cellular uptake and the final intracellular fate of CDplexes obtained with 2, 3 or 4 in COS-7 (African green monkey kidney fibroblasts used as MMR- and ASGPr-devoid controls) and BNL-CL2 cells using a set of selective endocytic pathway inhibitors in combination with fluorescently-labelled DNA. The main issues we have tried to uncover are whether the presence of glucopyranosyl or mannopyranosyl motifs in the vector has consequences in the preferred cell uptake mechanisms at work into MMR- and ASGPr-devoid cells and into cells expressing ASGPr lectins, specific for galactopyranosyl motifs, and above all, whether these preferred mechanisms are dependent on the cell type and on the nature of the peripheral sugar. Last, but not least, we aim at unequivocally establishing the involvement of the ASGPr in the uptake of GluCDplexes from gpaCD prototype 3 in BNL-CL2 hepatocytes and demonstrating an interaction of ASGPrs with the multivalent 6-amino6 -deoxy- $\beta$-D-glucopyranosyl display at the GluCDplexe surface. We keep in mind that polycationic sugar derivatives, such as chitosan, generally exhibit no or very low immunogenicity, making then very well-suited for their incorporation in biomaterials, ${ }^{35}$ and a similar behaviour should be expected for gpaCDformulated nanocomplexes.
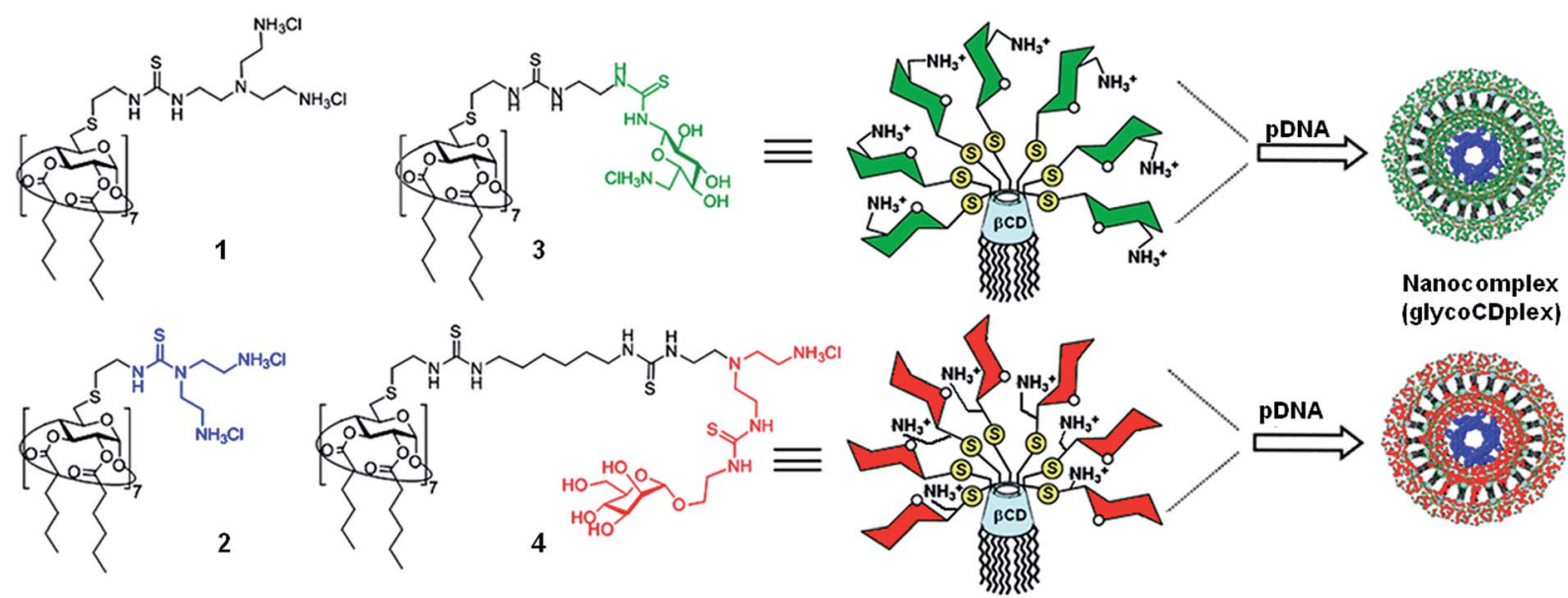

Fig. 1 Chemical structure of the polycationic amphiphilic cyclodextrin ( $\mathrm{paCD}$ ) vectors 1 and 2, and of the glycosylated polycationic amphiphilic cyclodextrins (gpaCDs) 3 and 4. A schematic representation of the formation of the glycosylated-decorated nanocomplexes 3:pDNA (GluCDplexes, with 6-amino-6-deoxy- $\beta$-D-glucopyranose residues, in green) and 4:pDNA (ManCDplexes, with $\alpha$-D-mannopyranosyl residues, in red) is shown. 


\section{Materials and methods}

\section{Materials}

The paCD derivative 2 (ref. 16) and the gpaCD derivatives 3 and 4 (ref. 22) were prepared according to previously reported procedures. JetPEI used as control was purchased from Polyplus SA (Illkirch, France). Chlorpromazine (CHZ), genistein (GNT) and amiloride (AML) were purchased from Sigma Aldrich. The YOYO-1 dye came from Invitrogen.

\section{Preparation of complexes formulated from CD derivatives and plasmids}

The plasmid pTG11236 (pCMV-SV40-luciferase-SV40 pA) used for the preparation of the DNA complexes as well as for the transfection and uptake assays is a plasmid of 5739 base pairs (bp). The pgWIZ-greeen fluorescent protein, pgWIZ-GFP, (pCMV-Intron-GFP) (5757 bp) plasmid (Aldevron) was also used for additional transfection experiments. For the uptake studies and prior to its formulation as CDplexes, the plasmid was first labelled with the fluorescent probe YOYO-1 (Molecular Probes, Eugene, USA) by addition of $1 \mu \mathrm{L}$ of YOYO- 1 stock solution ( $1 \mathrm{mM}$ in DMSO) to $100 \mu \mathrm{L}$ of a pDNA solution containing $1 \mu \mathrm{g} \mu \mathrm{L}^{-1}$ of pDNA in $20 \mathrm{mM}$ HEPES buffer ( $\mathrm{pH} 7.4$ ). This corresponds to a theoretical labelling density of 1 dye per $300 \mathrm{bp}$. The quantities of compound used were calculated according to the desired DNA concentration of $0.02 \mathrm{mg} \mathrm{mL}$ (i.e., $60 \mu \mathrm{M}$ phosphate), the $\mathrm{N} / \mathrm{P}$ ratio of 10 , the molar weight, and the number of protonable amine nitrogen atoms in the selected paCD 2 or pGaCD derivative or in JetPEI. For the preparation of the DNA complexes from CD derivatives, pDNA was diluted in HEPES ( $20 \mathrm{mM}, \mathrm{pH} 7.4)$ to a final concentration of $60 \mu \mathrm{M}$, and then the desired amount of CD derivative was added from a $10 \mathrm{mM}$ stock solution (DMSO). For JetPEI polyplexes, DNA was diluted in a $150 \mathrm{mM} \mathrm{NaCl}$ solution to a final phosphate concentration of $60 \mu \mathrm{M}$, and then the desired amount of JetPEI was added from a $7.5 \mathrm{mM} \mathrm{NaCl}$ solution (prepared from a $0.1 \mathrm{M}$ stock solution $\left(\mathrm{H}_{2} \mathrm{O}\right)$ ). The preparations were vortexed for $2 \mathrm{~h}$ and used for particle size measurements, transfection or uptake experiments.

\section{Measurement of the size of the complexes by dynamic light scattering (DLS)}

The average sizes of the CDplexes were measured using a Zetasizer nano (Malvern Instruments, Paris, France) with the following specification: sampling time, automatic; number of measurements, 3 per sample; medium viscosity, $1.054 \mathrm{cP}$; refractive index, 1.33; scattering angle, $173^{\circ} ; \lambda=633 \mathrm{~nm}$; temperature, $25{ }^{\circ} \mathrm{C}$. Data were analyzed using the multimodal number distribution software included in the instrument. Before each series of experiments, the performance of the instruments was checked with a standard sample of $90 \mathrm{~nm}$ monodisperse latex beads (Coulter). Results are given as volume distribution of the major population by the mean diameter with its standard deviation. No significant differences were encountered when the data were expressed in intensity or number distributions, whish is consistent with an spherical topology of the nanoparticles.

\section{Agarose gel electrophoresis}

Each CD derivative/DNA sample ( $20 \mu \mathrm{L}, 0.4 \mu \mathrm{g}$ of plasmid) was submitted to electrophoresis for about 30 min under $150 \mathrm{~V}$ through a $0.8 \%$ agarose gel in $1: 1: 1$ tris(hydroxymethyl)aminomethane (tris)/acetate/ethylenediaminetetraacetic acid (EDTA) buffer (TAE buffer) and stained by ethidium bromide ( $1 \mu \mathrm{L}$ of a $10 \mathrm{mg} \mathrm{mL}^{-1}$ solution for $20 \mathrm{~mL}$ of gel). DNA was then visualized after photographing using an UV transilluminator.

\section{Cell cultures}

COS-7 cells (African green monkey kidney fibroblast cell line transformed by an origin-defective mutant of SV40 coding for wild-type $\mathrm{T}$ antigen) were grown in Dulbelcco modified Eagle culture medium (DMEM; Gibco-BRL) containing 10\% v/v fetal calf serum (FCS; Sigma), glucose $\left(4.5 \mathrm{~g} \mathrm{~L}^{-1}\right)$, glutamine $(2 \mathrm{mM})$, penicillin (100 units per $\mathrm{mL}$ ), $100 \mu \mathrm{g} \mathrm{mL}^{-1}$ streptomycin in a wet $\left(37^{\circ} \mathrm{C}\right.$ ) and $5 \% \mathrm{CO}_{2} / 95 \%$ air atmosphere. BNL-CL2 cells (mouse normal embryonic liver cell line) were grown in DMEM containing $10 \%$ FCS, glucose $\left(4.5 \mathrm{~g} \mathrm{~L}^{-1}\right)$, glutamine $(2 \mathrm{mM})$, and $40 \mathrm{mg} \mathrm{mL}{ }^{-1}$ gentamycin in a wet $\left(37^{\circ} \mathrm{C}\right)$ and $5 \% \mathrm{CO}_{2} / 95 \%$ air atmosphere.

\section{Transfection assays}

Twenty-four hours before transfection, COS-7 or BNL-CL2 cells were seeded at a density of $2 \times 10^{4}$ cells per well in 96-well plates. The culture medium was then removed and replaced by $100 \mu \mathrm{L}$ of the above-described CDplexes in DMEM supplemented with $10 \%$ FCS so as to have $0.5 \mu \mathrm{g}$ of pDNA in the well (15 $\mu \mathrm{M}$ phosphate). After 4 and $24 \mathrm{~h}$, DMEM (50 and $100 \mu \mathrm{L}$ ) supplemented 10\% FCS, respectively, were added. After $48 \mathrm{~h}$, the transfection was stopped, the culture medium was discarded, and the cells were washed twice with PBS $(100 \mu \mathrm{L})$ and lysed with lysis buffer ( $50 \mu \mathrm{L}$; Promega, Charbonnières, France). The lysates were frozen at $-32{ }^{\circ} \mathrm{C}$ before the analysis of luciferase activity and of total cellular protein concentration. Measurement of luciferase activity was performed using a luminometer (GENIOS PRO, Tecan France S.A.) in dynamic mode, for $10 \mathrm{~s}$ on the lysis mixture $(20 \mu \mathrm{L})$ and using the "luciferase" determination system (Promega) in 96-well plates. The total protein concentration per well was determined by the BCA test (Pierce, Montluçon, France). Luciferase activity was calculated as femtograms ( $\mathrm{fg}$ ) of luciferase per $\mathrm{mg}$ of protein. The percentage of cell viability was calculated as the ratio of the total protein amount per well of the transfected cells relative to that measured for untreated cells $\times 100$. The data were calculated from three or four repetitions in two fully independent experiments (formulation and transfection).

\section{Kinetic studies}

After seeding and removal of the culture medium, cells were incubated for 2, 4, 6 and $24 \mathrm{~h}$ with $100 \mu \mathrm{L}$ of the above-described CDplexes in DMEM supplemented with $10 \%$ FCS so as to have 
$0.5 \mu \mathrm{g}$ of pDNA in the well $(15 \mu \mathrm{M}$ phosphate $)$. In the case of the $6 \mathrm{~h}$ incubation time experiment, DMEM $(50 \mu \mathrm{L})$ supplemented with $10 \%$ FCS was added to avoid discrepancy of cells. After each incubation times, the medium was discarded and the cells were successively placed for $15 \mathrm{~min}$ in a wet $\left(37^{\circ} \mathrm{C}\right)$ and $5 \% \mathrm{CO}_{2} /$ $95 \%$ air atmosphere with $500 \mu \mathrm{L}$ of a non supplemented DMEM solution containing calf thymus DNA $\left(200 \mathrm{mg} \mathrm{L}^{-1}\right)$ and then with $500 \mu \mathrm{L}$ of a non supplemented DMEM solution containing dextran sulphate sodium salt (mean MW $5 \times 10^{5} ; 200 \mathrm{mg} \mathrm{L}^{-1}$ ). The dextran sulphate medium was discarded and the cells were then washed 3 times with PBS $(500 \mu \mathrm{L})$. Finally, a set of these experiments was analyzed by flow cytometry to measure the mean fluorescence intensity (of 25000 cells) versus time incubation whereas another set was engaged in the transfection assays as described above. Flow cytometry was performed on FACScalibur (BD Biosciences) equipped with an argon laser $\left(\lambda_{\text {ex }}\right.$ $488 \mathrm{~nm}, \lambda_{\text {em }} 520 \mathrm{~nm}$ for YOYO and GFP). The software WinMDI 2.8 was used for the data analysis.

\section{Transfection and uptake assays by flow cytometry}

Twenty-four hours before transfection, COS-7 or BNL-CL2 cells were seeded at a density of $10^{5}$ cells per well in 24 -well plates. The culture medium was then removed and replaced by $500 \mu \mathrm{L}$ of the above-described YOYO-labelled pTG11236 or pWIZ-GFP CDplexes in DMEM supplemented with 10\% FCS so as to have $2.5 \mu \mathrm{g}$ of pDNA in the well (15 $\mu \mathrm{M}$ phosphate). After $4 \mathrm{~h}$ of incubation, the culture medium was discarded, and the cells were incubated for 15 min with $500 \mu \mathrm{L}$ of a calf thymus DNA solution $\left(200 \mathrm{mg} \mathrm{L}^{-1}\right)$ in non supplemented DMEM and in a wet $\left(37{ }^{\circ} \mathrm{C}\right)$ and $5 \% \mathrm{CO}_{2} / 95 \%$ air atmosphere. The cells were then washed twice with PBS $(500 \mu \mathrm{L})$ and detached with trypsin, and resuspended in $1 \mathrm{~mL}$ of DMEM supplemented with FCS. Cells were then centrifuged (5 min, $1200 \mathrm{rpm}$ ), washed with ice-cold PBS $(500 \mu \mathrm{L})$ then resuspended in PBS containing 1\% EDTA. The samples were then analyzed with a flow cytometer (same equipment and conditions as previously). The software WinMDI 2.8 was used for the data analysis.

\section{Assays in the presence of uptake inhibitors and control experiments}

Prior to incubation with the CDplexes, cells were pre-incubated with the culture medium containing chlorpromazine $(28 \mu \mathrm{M})$, genistein $(200 \mu \mathrm{M})$ or amiloride $(200 \mu \mathrm{M})$ for $1 \mathrm{~h}$ at $37^{\circ} \mathrm{C}$. The cell culture medium was then discarded and replaced by the CDplex solution, which contains fresh inhibitor at the same concentration. Incubation was then continued and treatment was performed as described above for the transfection and uptake studies. In order to confirm the suitability of these conditions to specifically inhibit cell uptake through the clathrin-mediated, caveolae-mediated or macropinocytosis routes, flow cytometry control experiments were performed using different endocytic fluorescent markers: $50 \mu \mathrm{g} \mathrm{mL}$ human transferrin-Alexa Fluor 594 conjugate (Molecular Probes), $1 \mu \mathrm{g} \mathrm{mL} \mathrm{m}^{-1}$ cholera toxin subunit B-Alexa Fluor A488 conjugate (Molecular Probes) and $1 \mathrm{mg} \mathrm{mL}^{-1} 70 \mathrm{kD}$ dextranFITC conjugate (Molecular Probes), respectively.

\section{Competition experiments in the presence of asialofetuin (ASF)}

To assess the implication of ASGPr recognition in CDplex internalization by BNL-CL2 cells, additional uptake and transfection studies were conducted in the presence of 1 and $5 \mathrm{mg} \mathrm{mL}^{-1}$ of asialofetuin (Sigma-Aldrich).

\section{Inhibition of uptake through the ASGPrs by blockage with ASGPr1 antibodies}

Preceding transfection, rabbit polyclonal antibodies to ASGPr1 (Abcam, Cambridge, UK) were applied to the cells in order to assess to which extent the uptake of gpaCDplexes is accounted for the ASGPrs. The cells were washed once in 2\% FBS in PBS and then unspecific binding was blocked by incubation with $2 \%$ goat serum in PBS at $37{ }^{\circ} \mathrm{C}$ for $30 \mathrm{~min}$. Then, the cells were incubated for another $2 \mathrm{~h}$ at $37{ }^{\circ} \mathrm{C}$ with $4 \mu \mathrm{g}$ of ASGPr1antibodies and washed twice with $2 \%$ FBS in PBS followed by transfection. This experiment was done in triplicate.

\section{Silencing of ASGPr gene in BNL-CL2 cells by RNA interference}

Silencing of ASGPr gene expression was facilitated using ASGPr1 gene-specific or scrambled (control) siRNA oligonucleotides (Santa Cruz Biotechnologies, Santa Cruz, CA) following the manufacturer's instruction. Briefly, BNL-CL2 cells were incubated as indicated in section 2.4 until $60-80 \%$ confluency, then transfected according to the manufacturer's protocol and, after addition of fresh medium, further cultured for $48 \mathrm{~h}$ prior to CDplex internalization or transfection studies. The level of ASGPr mRNA was evaluated by RT-PCR. The control siRNAs contain a scrambled sequence that does not lead to the specific degradation of any known cellular mRNA.

\section{Statistical analysis}

Statistical tests were performed with STATGRAPHICS Plus 5.0 software. Analysis of variance (Anova) was run on the logarithmic transformation of transfection levels (log 10 [fg luciferase per mg protein]) and on the cell viability to fit normal distributions of the data. Two factors, that is, the nature of the complexing agent (CD derivative and PEI) and the N/P ratio, were analyzed as the source of the variation of logarithmic transformation of the transfection levels and of cell variability percentages using a multiple comparison procedure. Tukey's honestly significant difference (HSD) method was used to discriminate among the means of cell viability percentages and the logarithmic transformation of luciferase expression levels.

\section{Results and discussion}

\section{CDplex characteristics}

The main goal of the present work was to analyze the uptake mechanism of three distinct cationic CDplex formulations in BNL-CL2 and COS-7 cells. Although the first interaction between the positively charged particles and the cell surface proteoglycans is probably electrostatic, the internalization mechanism can be severely affected by the physical features of the CDplexes, such as surface charge and size. ${ }^{36}$ The three 
paCDs 2-4 were shown previously to complex efficiently pDNA (pTG11236; luciferase encoding) at protonable nitrogen/ phosphorous ratio (N/P) 10, forming tiny spherical cationic nanoparticles that exhibited virtually identical size (mean hydrodynamic diameter ranging from 70 to $100 \mathrm{~nm}$ ), $\zeta$-potential (between +40 and $+50 \mathrm{mV}$ ) and ultrastructure as observable by transmission electron microscopy (TEM), ${ }^{16,22}$ which was confirmed in the present work. In the presence of $10 \%$ serum, their sizes increased to $120-150 \mathrm{~nm}$, pDNA remaining fully complexed and not accessible to ethidium bromide intercalation. These N/P 10 formulations were chosen keeping in mind that the homogeneity of their physico-chemical properties at the nanoscale should prevent differences in nanoparticle topology affecting significantly the preferred cell uptake mechanisms.

\section{Quantitative uptake studies}

Uptake kinetic studies of the N/P 10 CDplexes in BNL-CL2 or COS-7 cells were next performed by incubating the cells with CDplexes obtained from YOYO-1 labelled pDNA and the corresponding vector (2-4) at $37{ }^{\circ} \mathrm{C}$. FACS monitoring showed that the mean fluorescence associated to the cells reached a plateau after 2-3 h of incubation (data not shown). A 4 h-incubation period was then considered sufficient to warrant maximum uptake of the CDplexes by cells. It should further be stressed that no uptake by BNL-CL2 or COS-7 cells occurred with any of the CDplexes formulated with paCDs 2-4 when the experiments were conducted at $4{ }^{\circ} \mathrm{C}$, supporting that internalization of the CDplexes at $37{ }^{\circ} \mathrm{C}$ is controlled by an energy-dependent endocytic process. ${ }^{37}$

\section{GFP expression kinetics}

The GFP expression in BNL-CL2 cells for CDplexes formulated from paCD 2 in the presence of $10 \%$ serum as a function of incubation time, as compared with JetPEI N/P 10 polyplexes and naked pDNA are presented in Fig. 2. The mean fluorescence intensity of 2/pDNA CDplexes, JetPEI/pDNA

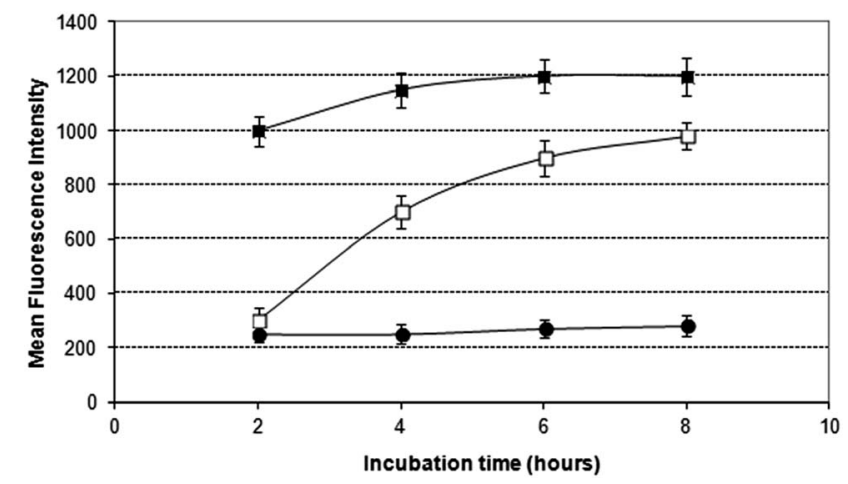

Fig. 2 GFP expression in BNL-CL2 cells after $2 \mathrm{~h}, 4 \mathrm{~h}, 6 \mathrm{~h}$ and $24 \mathrm{~h}$ of incubation with the CDplexes from paCD 2 (white squares) or JetPEI polyplexes (black squares) formulated at N/P 10 or naked pDNA (black circles) and in the presence of $10 \%$ serum. Data are presented as average \pm SD $(n=3)$. polyplexes or naked pDNA encoding for the GFP (labelled with the YOYO-1 green DNA dye) was monitored by flow cytometry after various incubation times (Fig. 2). The rapid internalization of JetPEI polyplexes was confirmed by the significant proportion of polyplexes that was taken up after only 2 and $4 \mathrm{~h}$ incubation times (83\% and $96 \%$ of the maximum value reached at the plateau, respectively). As for 2/pDNA CDplexes, the internalization is more progressive since the mean fluorescence intensity reached $30.6 \%, 71.4 \%$ and $92 \%$ of the maximum value after 2,4 and $6 \mathrm{~h}$ incubation times.

\section{Luciferase expression kinetics}

The luciferase expression data in BNL-CL2 cells for CDplexes formulated from paCD 2 in the presence of 10\% serum as a function of incubation time, as compared with JetPEI N/P 10 polyplexes, are presented in Fig. 3. While luciferase expression mediated by JetPEI was found to reach a plateau after $2 \mathrm{~h}$, longer periods of incubation were required to obtain a significant luciferase expression level in the case of CDplexes obtained from paCD 2. These data indicate that the uptake and intracellular trafficking of JetPEI polyplexes in BNL-CL2 cells, or of the intracellular vesicles containing these polyplexes from their uptake until penetration of pDNA into the cell nucleus, occurs much faster than for CDplexes. Most likely, these results also reflect that the corresponding DNA nanocomplexes are taken up by the BNL-CL2 cells through distinct mechanisms.

The rapid luciferase expression obtained with JetPEI in our experiments suggests that internalization of the corresponding polyplexes takes place mainly through the clathrin-coated pits into endosomes, as already found in other hepatocyte cell lines, ${ }^{38}$ being then released into the cytoplasm through the proton-sponge mechanism after endosome acidification. ${ }^{2}$ Indeed, it is known that the uptake kinetics of the clathrin pathway proceeds much faster than that of the caveolin route, ${ }^{39}$ the latter being the most productive one for cyclodextrin-based pDNA nanocomplexes in Vero cells. ${ }^{30}$ In any case, the kinetics data indicate that the $4 \mathrm{~h}$-incubation period we used for all assays was sufficient to warrant optimal gene expression with the CDplexes.

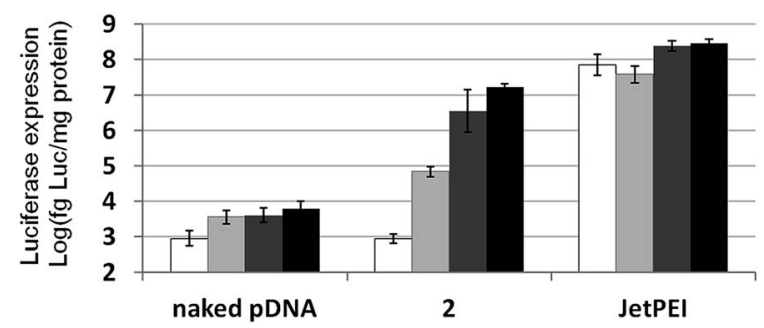

Fig. 3 Luciferase expression in BNL-CL2 cells after $2 \mathrm{~h}$ (white bar), $4 \mathrm{~h}$ (grey bar), $6 \mathrm{~h}$ (dark grey bar) and $24 \mathrm{~h}$ (black bar) of incubation with the CDplexes from paCD 2 or JetPEI polyplexes formulated at N/P 10 and in the presence of $10 \%$ serum. Data are presented as average \pm SD of three separate experiments. 
Endocytosis of CDplexes and cell viability in the absence and presence of inhibitors

To gain an insight into the relative contribution of the different uptake pathways in transfection of BNL-CL2 and COS-7 cells mediated by the N/P 10 CDplexes formulated from 2, 3 (GluCDplexes) or 4 (ManCDplexes), internalization of particles was monitored in the presence of three specific inhibitors of endocytic routes, namely: (i) chlorpromazine (CHZ), which inhibits the clathrin-dependent pathway related to coated pit formation, (ii) genistein (GNT), which inhibits the caveolamediated pathway related to recruitment of dynamin II and to disruption of actin network, and (iii) amiloride (AML), which inhibits macropinocytosis by the $\mathrm{Na}^{+} / \mathrm{H}^{+}$exchange proteins located at the cellular membranes. ${ }^{40}$ The drugs were utilized at $28 \mu \mathrm{M}$ for $\mathrm{CHZ}$ and $200 \mu \mathrm{M}$ for GNT or AML, in line with other studies where these concentrations were also found to have no significant impact on cell viability upon treatment with DNA nanocomplexes. ${ }^{41}$ In order to be able to correlate the transfection results with the operating internalization routes, identical conditions to those further employed for transfection studies $(10 \%$ serum) were used. Although a significant lose of efficiency has been reported for some of these inhibitors in the presence of serum, in our hands CHZ, GNT and AML at the indicated concentrations strongly reduced transferring, transferrin, cholera toxin and $70 \mathrm{kD}$ dextran uptake, respectively (58 to $65 \%$ inhibition; data not shown), in BNL-CL2 and COS-7 cells without promoting cell detachment, in agreement with the drug specificities and the known internalization mechanisms of the transferrin, the toxin and the polysaccharide (see Materials and methods section). Other studies ${ }^{39,42}$ also report efficient inhibitions in similar conditions (COS-7 cells with 10\% serum treated with inhibitors). The apparent discrepancy on the efficiency of the endocytic route inhibitors in the presence of serum in the literature, ascribed to the possible association of the drugs with serum proteins, ${ }^{43}$ could be due to differences in cell type and/or be related to altered characteristics of the cells after successive growing paths. Cell viability experiments (BCA test) in the presence of the inhibitors and of the corresponding N/P 10 CDplexes, used at a pDNA phosphate concentration of $60 \mu \mathrm{M}$, additionally established the suitability of the concentrations used in our study. We found, that cell viability for both cell lines remained in most cases very high ( $\geq 90 \%)$, excepting for the ManCDplexes on BNL-CL2 cells, for which cell viability was $80 \%$ when treated with inhibitors (see Fig. 5 herein after).

The above conditions were applied to YOYO-labelled CDplexes for cell uptake studies by flow cytometry. Next, the cells were re-suspended and analyzed alive and un-fixed by flow cytometry. Fig. 4 collects the percentages of YOYO-positive cells determined by this method. In principle, internalization of the CDplexes, as of cationic DNA complexes in general, into adherent cells must be facilitated by electrostatic interactions with the anionic proteoglycans at the cell surface. Owing to the shielding effect of the sugar units, we expected a decrease of the cell uptake rate for the Glu- and Man-CDplexes as compared with the CDplexes obtained from 2 . This was actually the case in COS-7 cells and for the ManCDplexes in BNL-CL2 cells in the absence of inhibitor. Notwithstanding, the GluCDplexes were internalized in BNL-CL2 cells as efficiently as the CDplexes from the non-glycosylated vector 2 . The percentage of YOYO-positive cells was actually above 3-fold higher for GluCDplexes as compared with ManCDplexes. Although not always the case, differences in the cell uptake and intracellular fate of glyconanoparticles often arise from interactions of the glycosylated particles with molecules/receptors located at the cell membrane. ${ }^{44}$ The relatively high uptake rate of the GluCDplexes in BNL-CL2 cells can, thus, be consistent with the existence of specific recognition processes mediating cell endocytosis.

Uptake of YOYO-labelled CDplexes formulated from paCD 2 into BNL-CL2 or COS-7 cells was almost unaffected after treatment with $\mathrm{CHZ}$, whereas a significant reduction was observed after cell treatment with GNT $(45,31 \%)$ or with a combination of CHZ and GNT $(64,52 \%)$, and, to a lesser extent, with AML (21, $18 \%)$. These data indicate that the uptake of the CDplexes from 2 into both cell lines does not involve clathrin-coated pit formation but is dependent to a great extent on the caveolaemediated endocytic pathway regulated by the recruitment of dynamin II, and to a lesser extent, on liquid-phase macropinocytosis.

Similarly, the percentage of BNL-CL2 or COS-7 cells that internalized Glu- or ManCDplexes (Fig. 4), was systematically reduced after cell treatment with GNT, indicating that caveolinmediated uptake probably plays a major role. The degree of cell uptake inhibition was above 2- to 3-fold higher in COS-7 (67, $75 \%$ ) as compared with BNL-CL2 cells (18, 33\%), however, suggesting that alternative internalization routes contribute at a larger extent in the later case. Unexpectedly, blocking the clathrin-dependent pathway with $\mathrm{CHZ}$ did not lead to reduction in CDplex uptake but to an important increase in the internalization rate. This result points to a certain degree of redundancy in the uptake routes of the sugar-decorated CDplexes. Similar "compensatory uptake" after inhibition of a given endocytic pathway has been demonstrated by other groups before. ${ }^{45}$ Actually, when the internalization experiments were conducted with a mixture of $\mathrm{CHZ}$ and GNT a strong uptake inhibition was observed in COS-7 cells (81, 87\%), which supports that both the clathrin and caveolin-mediated mechanisms act concomitantly and redundantly, and are the main internalization routes in this cell line. In contrast, the combination of $\mathrm{CHZ}$ and GNT resulted in a much lower decrease in uptake for BNL-CL2 cells $(28,37 \%)$, only slightly higher as compared with the inhibitory effect of GNT alone (Fig. 4). Alternative pathways must be active in this case for CDplex internalization. The weak effect of AML in the percentage of internalized cells $(0,21 \%)$ does not account for the experimental data, pointing to the participation of clathrinand caveolae-independent endocytic routes.

\section{Transfection of BNL-CL2 and COS-7 cells by CDplexes in the absence and presence of inhibitors}

Both uptake and intracellular routing of gene delivery systems are of major importance to achieve gene expression. The effects of blocking specific routes on gene expression efficiency in BNLCL2 and COS-7 were therefore determined by assaying 


\section{BNL-CL2}
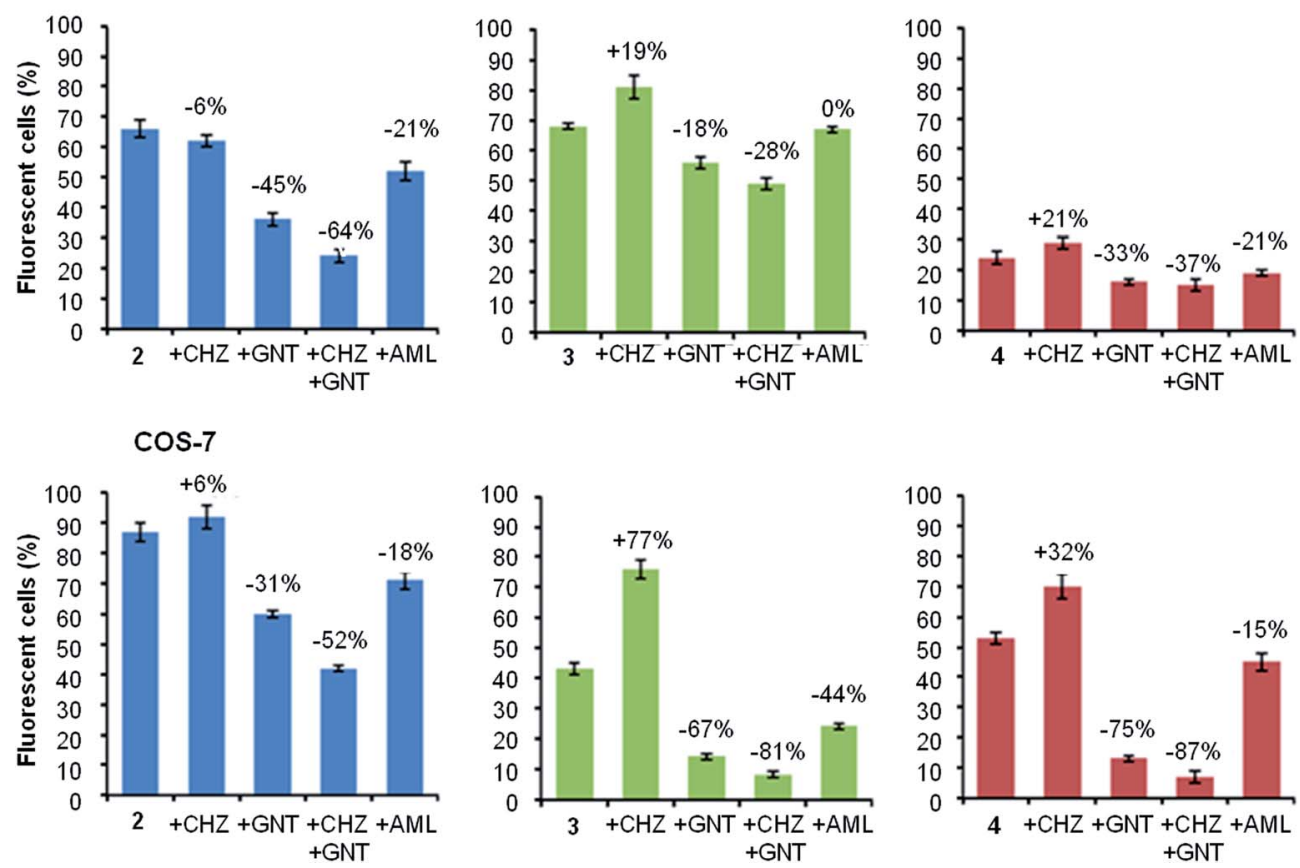

Fig. 4 Percentage of fluorescent BNL-CL2 (upper graphs) or COS-7 cells (lower graphs) obtained after transfection in serum (10\%) medium with N/P 10 YOYO-labelled pTG11436 CDplexes formulated from paCD 2 (blue bars), GluCD 3 (green bars) or ManCD 4 (red bars) in the absence or presence of internalization inhibitor drugs $(28 \mu \mathrm{M} \mathrm{CHZ}, 200 \mu \mathrm{M}$ GNT, $200 \mu \mathrm{M} \mathrm{AML})$ as determined by flow cytometry. The data are presented as average \pm SD of two separate experiments. The data over the bars represent the percentage of uptake inhibition (-) or enhancement (+) with respect to untreated cells.

luciferase (pTG11436 plasmid, Fig. 5) or green fluorescent protein (GFP; pgWIZ-GFP plasmid) $48 \mathrm{~h}$ after transfection with the CDplexes. The inhibitors themselves did not negatively influence gene expression. Consequently, decrease in transgene expression can be ascribed to the effect of inhibitors on uptake and/or intracellular routing.

For the non-glycosylated CDplexes formulated from paCD 2, the observed transfection data qualitatively correlated with the results discussed above on the predominance of the caveolaemediated and, to a lesser extent, macropinocytosis cell uptake pathways. Thus, in both cell lines luciferase expression remained virtually unchanged in the presence of $\mathrm{CHZ}$ but decreased by 10- to 100-fold in the presence of GNT and by 5- to 10-fold in the presence of AML. Transfection data obtained for pgWIZ-GFP CDplexes in BNL-CL2 hepatocytes in the absence and presence of CHZ, GNT and AML followed essentially the same trends encountered with pTG11236 CDplexes, but transfection efficiency was generally lower. Thus, for pgWIZ-GFP:2 CDplexes, only $18 \%$ of the cells expressed GFP after the transfection assay in the absence of any inhibitor, to be compared with $66 \%$ in the case of pTG11236:2 CDplexes. Variations by the action of the inhibitors were $-6 \%(\mathrm{CHZ}),-44 \%$ (GNT), $-67 \%$ $(\mathrm{CHZ}+\mathrm{GNT})$ and $+6 \%$ (AML), to be compared with $-6 \%,-45 \%$, $-64 \%$ and $-21 \%$, respectively. The caveolin-mediated mechanism of CDplex cellular uptake is thus the most efficient one in terms of transfection.

ManCDplexes and GluCDplexes behaved similarly to the non-glycosylated CDplexes from 2 in COS-7 cells, meaning that the fraction of sugar-decorated nanocomplexes uptaken via clathrin-mediated endocytosis is essentially unproductive regarding transfection. A totally different scenario was encounter when assayed in BNL-CL2 cells: transfection was enhanced in the case of GluCDplexes in the presence of any of the three inhibitors, the effect being particularly notable in the case of $\mathrm{CHZ}$, whereas it remained unchanged in the case of ManCDplexes (Fig. 5).

It must be noted that efficient cell uptake of DNA complexes is not necessarily correlated with an efficient expression into the cell of the DNA-encoded protein, which is a complex phenomenon depending among others upon the nature of the endocytic vesicles containing the DNA nanoparticles, their intracellular fate en route to the nucleus, and the delivery of the cargo (gene) into the nucleus. In the present study, GluCDplexes were the most efficiently uptaken by BNL-CL2 cells (Fig. 4), but the transfection efficiency was drastically poorer (5-to-3 orders of magnitude; Fig. 5) than for the non-glycosylated CDplexes from 2 or for the ManCDplexes. This result strongly suggests that a non-productive internalization route is favored in this case. The dramatic increase in protein expression in the presence of $\mathrm{CHZ}$, near 4 orders of magnitude, can be rationalized assuming that the clathrin-mediated pathway is responsible for the nonproductive internalization of the GluCDplexes and that redundancy of uptake mechanisms results in CDplex internalization by alternative productive routes upon clathrin-coated pit formation inhibition. The fact that transfection efficiency is also enhanced, although to a lesser extent, in the presence of GNT 
BNL-CL2

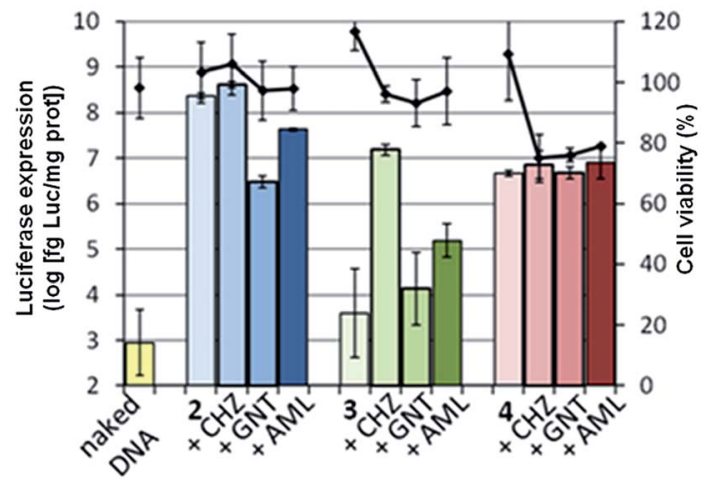

cos-7

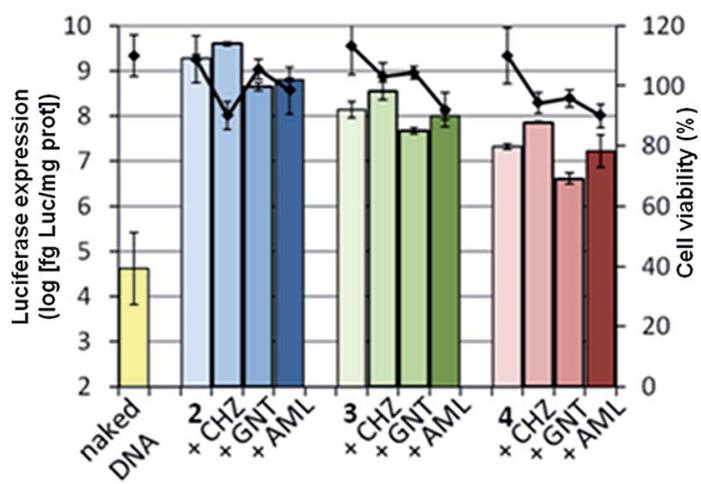

Fig. 5 In vitro gene transfection efficiency (left axis; bars) and cell viability (right axis; squares and lines) in BNL-CL2 (upper graph) and COS-7 cells (lower graph) of CDplexes prepared from paCD 2 (blue bars), GluCD 3 (green bars) or ManCD 4 (red bars) at N/P 10 in the absence or presence of $28 \mu \mathrm{M}$ chlorpromazine ( $\mathrm{CHZ}$ ), $200 \mu \mathrm{M}$ genistein (GNT) or $200 \mu \mathrm{M}$ amiloride (AML). Naked DNA (yellow bar) was used as negative control.

and AML discards that caveolae-mediated endocytosis or macropinocytosis are the only uptake mechanisms leading to protein expression and suggests that productive clathrin- and caveolae-independent routes are responsible, at least in part, for the observed compensatory effect. Productive clathrin- and caveolae-independent uptake seems to be also relevant for the ManCDplexes, compensating for the decrease in CDplex internalization via the caveolae endocytic mechanisms in the presence of GNT.

\section{Involvement of ASGPr in endocytosis of GluCDplexes by BNL- CL2 cells}

It is well-established that ASGPr-mediated internalization proceeds through the clathrin pathway, ${ }^{46}$ which was recently found to trigger non-productive internalization in the case of galactosylated CDplexes in HepG2 cells. ${ }^{30}$

To ascertain whether GluCDplexes formulated from 3 are likewise internalized in BNL-CL2 hepatocytes via the mechanism that involves ASGPr-dependent recognition of galactose residues in asialoglycoproteins, asialofetuin (ASF) was included at 1 and $5 \mathrm{mg} \mathrm{mL}^{-1}$ concentration as a soluble competitive ligand during the incubation of the cells with the CDplexes for internalization and transfection studies. As shown in Fig. 6, inclusion of ASF significantly $(P<0.01)$ decreases uptake of YOYO-labelled GluCDplexes in BNL-CL2 cells in a dosedependent manner (Fig. 6A), but with a concomitant enhancement in transfection efficiency (Fig. 6B). A very similar set of data was obtained when the receptors were blocked with antiASGPr1 antibodies prior to internalization assessment and transfection with luciferase-encoding pDNA. For comparison, ASF or anti-ASGPr1 antibody treatment had no effect in COS-7 cell transfection promoted by GluCDplexes or in transfection mediated by the non-glycosylated CDplexes from 2 or the ManCDplexes from 4 in BNL-CL2 or COS-7 cells (data not shown). The decrease in GluCDplexes' uptake by BNL-CL2 cells in the presence of ASF and anti-ASGPr1 antibody is consistent with the presumed implication of ASGPrs in endocytosis. Contrary to that observed when the clathrin route was fully inhibited with $\mathrm{CHZ}$, compensation by other cell entry routes did not balance entirely the effect of ASGPr blocking, which is in agreement with the assumption that the much higher uptake of the GluCDplexes as compared with the ManCDplexes in hepatocytes results from specific recognition between the multivalent 6-aminoglucose peripheral display and ASGPrs. The dramatic increase in luciferase expression following ASF or antiASGPR antibody treatment for GluCDplexes probably results from activation of alternative endocytic pathways that, though less efficient regarding uptake, would be much more productive in terms of transfection. Caveolae-mediated and clathrin and

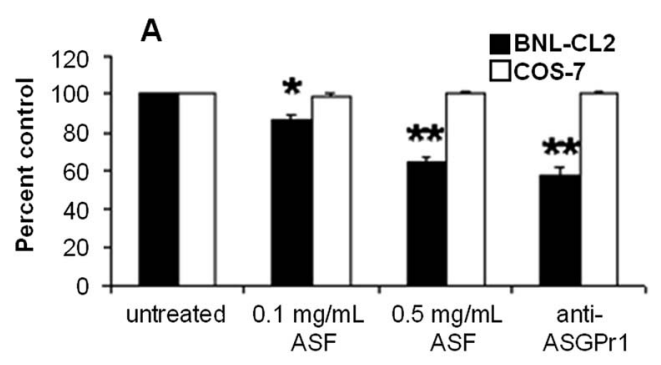

B

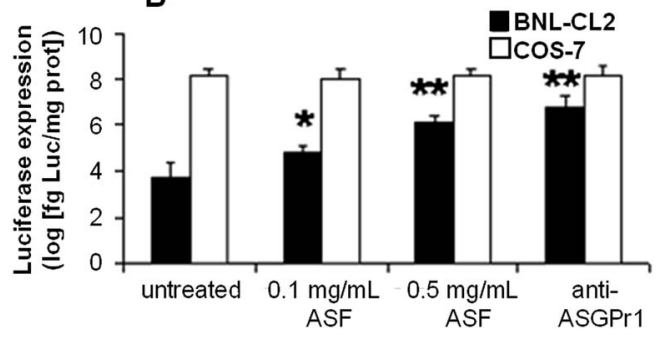

Fig. 6 Effects of ASGPr blocking with asialofetuin (ASF, 0.1 and $0.5 \mathrm{mg}$ $\mathrm{mL}^{-1}$ ) and ASGPr1-antibodies on cell uptake (A) and transfection efficiency (B) of N/P 10 GluCDplexes formulated with 3 in BNL-CL2 or COS-7 cells. (A) Ratio of fluorescent cells obtained after transfection in serum (10\%) medium with YOYO-labelled pTG11436 GluCDplexes; data are presented as average relative to control \pm SD of two separate experiments. (B) Luciferase expression after transfection with GluCDplexes formulated with the luciferase-encoding reporter plasmid pTG11236; data are presented as average \pm SD $(n=3)$. $* P<$ 0.01. $* * P<0.001$ 

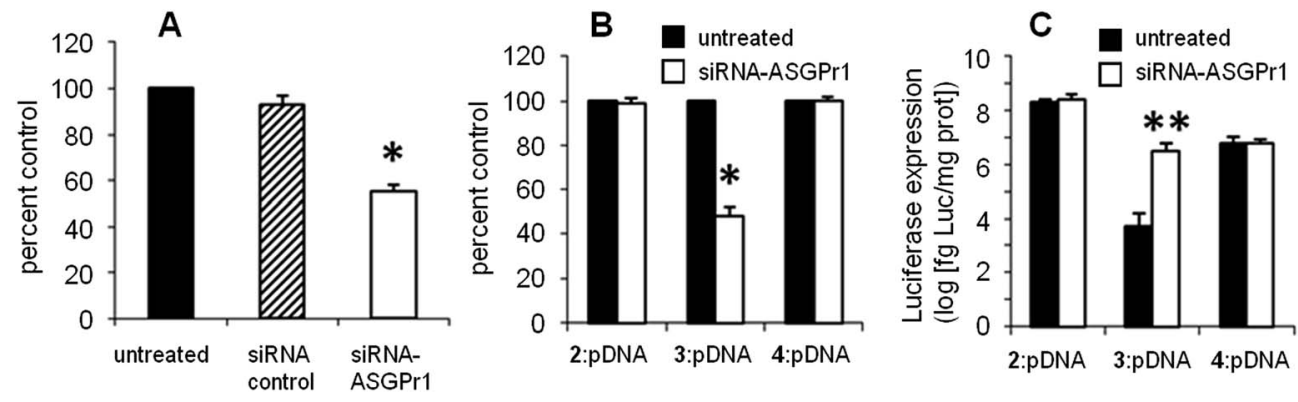

Fig. 7 Effects of siRNA-mediated ASGPr1 gene knockdown in cell uptake and transfection efficiency in BNL-CL2 hepatocytes of N/P 10 pTG11436 CDplexes. (A) ASGPr1 gene detection levels following siRNA treatment as determined by RT-PCR; data represent the mean \pm SD relative to untreated BNL-CL2 cells ( $n=3$, two independent experiments). (B) Ratio of fluorescent siRNA-untreated and treated cells obtained after transfection in serum (10\%) medium with YOYO-labelled CDplexes from paCD 2, GluCD 3 and ManCD 4; data are presented as average relative to control \pm SD of two separate experiments. (C) Luciferase expression after transfection with CDplexesof siRNA-untreated and treated; data are presented as average $\pm \mathrm{SD}(n=3) . * P<0.001 . * * P<0.0001$

caveolae-independent internalization may account for this effect.

We sought to confirm a role of ASGPr in GluCDplex uptake into BNL-CL2 cells by way of ASGPr-specific RNA interference. As shown in Fig. 7A, transfection of BNL-CL2 cells with a siRNA sequence silencing ASGPr1 gene expression significantly $(P<$ 0.001) reduced the level of gene transcription as determined by real-time PCR (RT-PCR), while a scrambled siRNA control had no significant effect on ASGPr1 expression when compared with untreated controls. As illustrated in Fig. 7B and C, ASGPr1specific gene knockdown significantly reduced the internalization rate of fluorescently labelled GluCDplexes $(P<0.001)$ and increased substantially ( 3 orders of magnitude) luciferase expression $(P<0.0001)$, respectively. By contrast, ASGPr1specific gene knockdown did not affect the internalization rate or the transfection efficiency of the non-glycosylated CDplexes from 2 or ManCDplexes.

The above results firmly demonstrate that ASGPr is involved in target recognition and internalization of the glucopyranosyldecorated GluCDplexes by BNL-CL2 cells and that suppression of this uptake route activates alternative uptake mechanisms that are much more productive in terms of transfection (Fig. 7C). They also suggest that the 6-amino-6-deoxy- $\beta$-D-glucopyranosyl residue, at least when presented in a multivalent manner at the GluCDplexe's surface, behaves as a functional mimic of D-galactose towards ASGPr, allowing hepatocyte targeting. A preliminary enzyme-linked lectin assay (ELLA) experiment indicated that GluCDplexes from 3 efficiently inhibit binding of the galactose specific lectin peanut agglutinin (PNA) to a galactosylated polymer, ${ }^{47-49}$ further supporting this notion (data not shown). Analogous competitive experiments using the ASGPr and different multivalent 6-amino-6-deoxy- $\beta$-D-glucopyranosyl should confirm this point. Work in that direction is currently sought in our laboratories.

\section{Conclusions}

The ensemble of data for the new CDplexes formulated from the polycationic amphiphilic $\beta C D$ derivative 2 and the glucosylated
3 and mannosylated 4 congeners demonstrated that the presence of a multivalent carbohydrate display at the glycoCDplex surface has a strong influence in the uptake mechanisms and the transgene expression. Different cell types showed distinct preferences in respect to the mechanisms that they make use of to internalize the glycoCDplexes, these preferences being also dependent on the nature of the sugar ligands present at the surface of the nanoparticles. Caveolin-mediated endocytosis was the most relevant pathway both in BNL-CL2 and COS-7 cells for the non-glycosylated vector 2 , leading to efficient transgene expression. This was also the main productive route for Gluand ManCDplexes from gpaCDs 3 and 4, respectively, but it coexisted in these cases with clathrin-mediated (nonproductive) as well as clathrin- and caveolin-independent endocytosis (productive), drawing a rather complex scenario where blocking of an uptake route is compensated by alternative pathways. The contribution of the clathrin route in the BNLCL2 cell line was particularly significant for the 6-amino-6deoxy- $\beta$-D-glucopyranosyl gpaCD 3 due to most probably ASGPrmediated uptake of the corresponding GluCDplexes into clathrin-coated endosomes. In this particular context, it would be most instructive to examine and compare the uptake mechanisms and fate (i) of the Glu- and ManCDplexes into other ASGPr-expressing cell lines, and (ii) of cationic aminogalactopyranosyl-appended CDplexes, which are expected to exhibit higher affinity towards ASGPr than the aminoglucopyranosyl congeners. In a more general context, using cationic lectin ligands for glycotargeting purposes is expected to be particularly interesting to avoid the often negative impact of neutral oligosaccharides in the membrane crossing capabilities of glycodecorated nanoparticles. ${ }^{32}$

\section{Conflict of interest}

The authors confirm that there are no known conflicts of interest associated with this publication and there has been no significant financial support for this work that could have influenced its outcome. 


\section{Acknowledgements}

NG, CB, CC and AD-M are grateful to the CNRS and Région Provence, Alpes, Côte d'Azur, to the Ministère de l'Enseignement Supérieur et de la Recherche (France) and to the CSIC (JAE program), respectively, for doctoral fellowships. This work was also supported by the Spanish Ministerio de Economía y Competitividad (contract numbers CTQ2010-15848 and SAF201344021-R) co-financed with the Fondo Europeo de Desarrollo Regional FEDER, the Junta de Andalucía, the CSIC, and FUSINT (CNR project). Technical support from CITIUS (Universidad de Sevilla) and from Agnès Loubat (IBV, UMR 7277, CNRS, Université de Nice Sophia Antipolis) is also acknowledge.

\section{References}

1 M. Giacca, Gene Therapy, Springer-Verlag, Milan, 2010.

2 J. Nguyen and F. Szoka, Acc. Chem. Res., 2012, 45, 1153-1162.

3 M. Giacca and S. Zacchigna, J. Controlled Release, 2012, 161, 377-388.

4 J. P. Behr, Acc. Chem. Res., 2012, 45, 980-984.

5 M. A. Mintzer and E. E. Simanek, Chem. Rev., 2009, 109, 259302.

6 R. Srinivas, S. Samanta and A. Chaudhuri, Chem. Soc. Rev., 2009, 38, 3326-3338.

7 S. Bhattacharya and A. Bajaj, Chem. Commun., 2009, 46324656.

8 N. Surendra, G. Nidhi and C. Ramesh, J. Biomed. Nanotechnol., 2011, 7, 504-520.

9 D. Putnam, Nat. Mater., 2006, 5, 439-451.

10 C. Ortiz Mellet, J. M. Benito and J. M. García Fernández, Chem.-Eur. J., 2010, 16, 6728-6742.

11 D. Sigwalt, M. Holler and J. Iehl, et al., Chem. Commun., 2011, 4640-4642.

12 V. Bagnacani, V. Franceschi and L. Fantuzzi, et al., Bioconjugate Chem., 2012, 23, 993-1002.

13 R. V. Rodik, A. S. Klymchenko and N. Jain, et al., Chem.-Eur. J., 2011, 17, 5226-5238.

14 C. Ortiz Mellet, J. M. García Fernández and J. M. Benito, Chem. Soc. Rev., 2011, 40, 1586-1608.

15 A. Díaz-Moscoso, P. Balbuena and M. Gómez-García, et al., Chem. Commun., 2008, 2001-2003.

16 A. Díaz-Moscoso, L. Le Gourriérec and M. Gómez-García, et al., Chem.-Eur. J., 2009, 15, 12871-12888.

17 A. Méndez-Ardoy, N. Guilloteau and C. Di Giorgio, et al., J. Org. Chem., 2011, 76, 5882-5894.

18 C. Bienvenu, A. Martínez and J. L. Jiménez Blanco, et al., Org. Biomol. Chem., 2012, 10, 5570-5581.

19 A. M. O'Mahony, J. Ogier and S. Desgranges, et al., Org. Biomol. Chem., 2012, 10, 4954-4960.

20 A. Méndez-Ardoy, K. Urbiola and C. Aranda, et al., Nanomedicine, 2011, 6, 1697-1707.

21 N. Symens, A. Méndez-Ardoy and A. Díaz-Moscoso, et al., Bioconjugate Chem., 2012, 23, 1276-1289.

22 A. Díaz-Moscoso, N. Guilloteau and C. Bienvenu, et al., Biomaterials, 2011, 32, 7263-7273.
23 A. McMahon, M. J. O'Neill and E. Gomez, et al., J. Pharm. Pharmacol., 2012, 64, 1063-1073.

24 J. Guo, J. R. Ogier and S. Desgranges, et al., Biomaterials, 2012, 33, 7775-7784.

25 A. Martínez, C. Ortiz Mellet and J. M. García Fernández, Chem. Soc. Rev., 2013, 42, 4746-4773.

26 I. A. Khalil, K. Kogure and H. Akita, et al., Pharmacol. Rev., 2006, 58, 32-45.

27 L. Medina-Kauwe, J. Xie and S. Hamm-Alvarez, Gene Ther., 2005, 12, 1734-1751.

28 P. Midoux, G. Breuzard and J. P. Gomez, et al., Curr. Gene Ther., 2008, 8, 335-352.

29 M. J. Reilly, J. D. Larsen and M. O. Sullivan, Mol. Pharm., 2012, 9, 1280-1290.

30 A. Díaz-Moscoso, D. Vercauteren and J. Rejman, et al., J. Controlled Release, 2010, 146, 318-325.

31 H. Zhang, Y. Ma and X. L. Sun, Med. Res. Rev., 2010, 30, 270289.

32 C. Ortiz Mellet, J. M. García Fernández and J. M. Benito, Carbohydr. Chem., 2012, 38, 338-375.

33 M. Spiess, Biochemistry, 1990, 29, 10009-10018.

34 L. Desigaux, M. Sainlos and O. Lambert, et al., Proc. Natl. Acad. Sci. U. S. A., 2007, 104, 16534-16539.

35 F. Ding, H. Deng and Y. Du, et al., Nanoscale, 2014, 6, 94779493.

36 J. Rejman, V. Oberle and I. S. Zuhorn, et al., Biochem. J., 2004, 377, 159-169.

37 J. Saraste, G. E. Palade and M. G. Farquhar, Proc. Natl. Acad. Sci. U. S. A., 1986, 83, 6425-6429.

38 K. von Gersdorff, N. N. Sanders and R. Vandenbroucke, et al., Mol. Ther., 2006, 14, 745-753.

39 M. A. E. M. van der Aa, U. S. Huth and S. Y. Häfele, et al., Pharm. Res., 2007, 24, 1590-1598.

40 D. Vercauteren, J. Rejman and T. F. Martens, et al., J. Controlled Release, 2012, 161, 566-581.

41 S. Xiang, H. Tong and Q. Shi, et al., J. Controlled Release, 2012, 158, 371-378.

42 U. S. Huth, R. Schubert and R. Peschka-Süss, J. Controlled Release, 2006, 110, 490-504.

43 K. Von Gersdorff, N. N. Sanders and R. Vandenbroucke, et al., Mol. Ther., 2006, 14, 745-753.

44 M. Moros, B. Hernáez and E. Garet, et al., ACS Nano, 2012, 6, 1565-1577.

45 Z. Rehman, I. S. Zuhorn and D. Hoekstra, J. Controlled Release, 2013, 166, 46-56.

46 L. M. Bareford and P. W. Swaan, Adv. Drug Delivery Rev., 2007, 59, 748-758.

47 M. Gómez García, J. M. Benito and A. P. Butera, et al., J. Org. Chem., 2012, 77, 1273-1288.

48 R. Lotan, E. Skutelsky and D. Danon, et al., J. Biol. Chem., 1975, 250, 8518-8523.

49 M. Almant, A. Mastouri and L. Gallego-Yerga, et al., Chem.Eur. J., 2013, 19, 729-738. 